\title{
Penetration Enhancing of an Erythrocyte-Mimicking Nanoplatform via Papaverine for Radiosensitization
}

\author{
Jinrui Zhang' \\ Huaicheng Wang' \\ Weilong Suo ${ }^{2}$ \\ Zhizhou Li' \\ Chunxu Yang ${ }^{3}$ \\ 'Department of Orthopedics, China- \\ Japan Union Hospital of Jilin University, \\ Changchun, 130033, People's Republic of \\ China; ${ }^{2}$ Key Laboratory of Applied \\ Chemistry and Nanotechnology at \\ Universities of Jilin Province, Changchun \\ University of Science and Technology, \\ Changchun, 130022, People's Republic of \\ China; ${ }^{3}$ Department of Radiation and \\ Medical Oncology, Hubei Key Laboratory \\ of Tumor Biological Behaviors, Hubei \\ Cancer Clinical Study Center, Zhongnan \\ Hospital of Wuhan University, Wuhan, \\ 43007I, People's Republic of China
}

Correspondence: Zhizhou Li; Chunxu

Yang

Email zhizhou@jlu.edu.cn;

youer9youer@I63.com
Purpose: Radiotherapy (RT) is recommended as an extensive therapeutic regimen for cancer patients; however, cancer radio-resistance results from reduced oxygen levels (hypoxia) in the tumor microenvironment. Herein, we report a therapeutic strategy that greatly enhances the treatment effects of RT.

Methods: Specifically, papaverine (ppv), an FDA-approved smooth muscle relaxant, was applied in the strategy. Ppv improved blood flow via vasodilation to deliver sufficient oxygen to the hypoxic solid tumor and further resulted in increased tumor penetration of the radiosensitizer, significantly enhancing the radiosensitization compared with no ppv treatment. Additionally, tantalum oxide nanospheres were cloaked in red blood cell membranes ( $\mathrm{TaOx} @ \mathrm{M})$ to achieve greater biocompatibility, non-immunogenicity, and a longer circulation time.

Results: As a high- $Z$ element, tantalum provides localized dose enhancement and thereby boosts the efficacy of RT. Vasodilation, the oxygenation of cancer cells, and the improved accumulation and retention of $\mathrm{TaOx} @ \mathrm{M}$ in the tumor region were verified in vivo. Furthermore, compared with RT alone, the combined vasodilation and nanosphere camouflaging strategy more efficiently suppressed the growth of K7M2 tumors in mice.

Conclusion: The results of this study suggest that the integration of TaOx@M and ppv has excellent potential for improving RT efficacy.

Keywords: radiotherapy, hypoxia, tantalum oxide, papaverine, vessel vasodilation

\section{Introduction}

Because of radio-resistance, radiotherapy (RT) is limited as a palliative regimen for osteosarcoma cancer and as a local control. ${ }^{1,2}$ At least one factor accounts for radioresistance: aberrant angiogenesis, which is due to the excessive proliferation of tumors cells, ${ }^{3}$ and the resulting lack of oxygen stabilizes the DNA double-strand breaks. $^{4-6}$ In this respect, methods of relieving hypoxia are attracting attention for improving the effectiveness of RT. ${ }^{7-10}$ Although, overall, the mechanisms of resistance to RT are complex and remain unclear.

In order to avoid acute or chronic radiation effects, it is crucial that we identify a therapeutic strategy to enhance the deposition of radiation energy at the tumor target and to alleviate side effects in adjacent tissues or vulnerable organs. ${ }^{11}$ To promote the sensitivity of cancer cells to radiation, the use of elements with high atomic numbers for generating photo/auger electrons has been recommended. ${ }^{12}$ For instance, gold, ${ }^{13,14}$ bismuth, ${ }^{15,16}$ platinum, ${ }^{17}$ and tantalum ${ }^{18,19}$ nanoplatforms have been assessed as radiosensitizers. Despite substantial recent advances, difficulties with enhancing radiosensitization remain. Important issues include increasing the 
ability of the radiosensitizer to penetrate the tumor and reach all viable cells and assisting the radiosensitizer to escape clearance by the reticuloendothelial system (RES). ${ }^{5,20-22}$

Nevertheless, most tumors tend to "outgrow" the blood supply as a result of rapid cellular proliferation. To meet the demand of cancerous cells for nutrition and oxygen, angiogenesis is stimulated and new primitive blood vessels are formed, resulting in large interstitial spaces among the vessels, which often results in cancer cells being distant from blood vessels. Within tumor interstitial spaces, the dense extracellular matrix comprises a hydrophilic gel with a high ratio of collagen and elastic fiber, proteoglycans, and glycosaminoglycan, and it has an elevated fluid pressure because of heterogeneity. ${ }^{23,24}$ Meanwhile, the primitive tumor vasculature is morphologically and functionally abnormal and causes disrupted blood flow. The large interstitial space, dense extracellular matrix, and abnormal angiogenesis in tumors decrease the nanoparticle diffusion rate. Meanwhile, these factors increase the distance nanoparticles have to travel, and hinder their penetration into solid tumors, causing intratumoral diffusion resistance which cause uneven distribution of radiosensitizers. ${ }^{21}$ The nanoparticle's limited ability to penetrate the tumor and access all tumor stem cells compromises the efficiency of the radiosensitizer, which can allow the tumor to regenerate and lead to recurrence. Therefore, attention should be given to improving nanoparticle penetration and cellular uptake in tumorous regions. ${ }^{25,26}$ Some drugs increase blood flow perfusion and, therefore, permeabilize tumor blood vessels to enhance the penetration and accumulation of nanoparticles. ${ }^{24,26-32}$ For example, iRGD peptide improves tissue permeability via $\alpha v$-integrin and neuropilin-1. ${ }^{27,31}$ Dexamethasone, with anti-inflammatory properties, increases tumor accumulation by degradation or normalization of the extracellular matrix in the interstitial space. ${ }^{31}$ The normalization of vascular abnormalities is also essential in intratumoral transport. ${ }^{33-35}$ When endowed with outstanding permeability, radiosensitizers have the capacity to access the tumor region and efficiently penetrate the cancerous site while reducing accumulation in undesired tissues and, subsequently, enhancing the therapeutic outcome of ionizing radiation.

In this study, we proposed a strategy to enhance the therapeutic effects of ionizing radiation by improving the tumor oxygenation status and enhancing radiosensitizer delivery (Scheme 1). Specifically, we employed ppv, a benzylisoquinoline alkaloid isolated from opium poppy (papaver somniferum), ${ }^{36}$ which is a clinically approved non-selective smooth muscle relaxant for blood vessels that, therefore, improve ischemia. ${ }^{37,38}$ In clinical trials, ppv administration has been validated as an agent that increases vessel perfusion. ${ }^{39,40}$ To improve the effectiveness of therapeutics, researchers have turned to nature for design ideas: the red blood cell (RBC) membrane, which is the most abundant natural carrier in the blood, ${ }^{41-44}$ was used to camouflage the radiosensitizer tantalum oxide $(\mathrm{TaOx})$, allowing it to escape immune attack. ${ }^{20,22,45-47}$ Therefore, we employed this strategy to enhance RT along with ppv-mediated vasodilation. Both photoacoustic (PA) imaging and immunohistochemistry analysis showed that tumor oxygen saturation was dramatically enhanced 1 $\mathrm{h}$ post-ppv injection, indicating that ppv can alleviate hypoxia in the tumor region by promoting the blood flow to the tumor. However, the permeability of tumor blood vessels can be modified to allow nanoparticles access to most cancerous cells and, hence, increase their efficacy, which was confirmed by further pharmacokinetic and TaOx biodistribution analysis. Hereby, on one hand, ppv improved the oxygen status in tumors, on the other hand, the penetration of $\mathrm{RBC}$ membrane cloaked $\mathrm{TaOx}$ was also modified. This therapy strategy shows potential for use in effectively inhibiting solid tumors by enhancing RT.

\section{Materials and Methods}

\section{Preparation of $\mathrm{TaOx}$ and $\mathrm{TaOx} @ \mathrm{M}$}

$\mathrm{TaOx}$ nanoparticles were synthesized as described in a previous report and all the sources of chemicals were shown in supporting information $1.1{ }^{48}$ For the synthesis of hollow TaOx nanospheres, briefly, $1 \mathrm{~mL}$ of deionized water and $0.1 \mathrm{~mL}$ of ethanol were added to a round-bottomed flask. Then, $4 \mu \mathrm{L}$ of tantalum ethoxide and $80 \mu \mathrm{L}$ of anhydrous ethanol were mixed until homogeneous, and the mixture was added to the flask in drops. After the mixture was stirred vigorously for $30 \mathrm{~min}$ at room temperature, it was centrifuged for $10 \mathrm{~min}$ at $10,000 \mathrm{rpm}$, and the supernatant was removed. The product was washed with deionized water several times and dried in a vacuum overnight at $40{ }^{\circ} \mathrm{C}$.

Whole blood was withdrawn from Balb/c mice (6-8 weeks old) and stored in EDTA blood collection tubes with ethylenediamine tetraacetic acid $(1.5 \mathrm{mg} / \mathrm{mL})$ as an anticoagulant. The RBC vesicles were obtained using the low-osmosis method. ${ }^{49}$ The blood was centrifuged at 


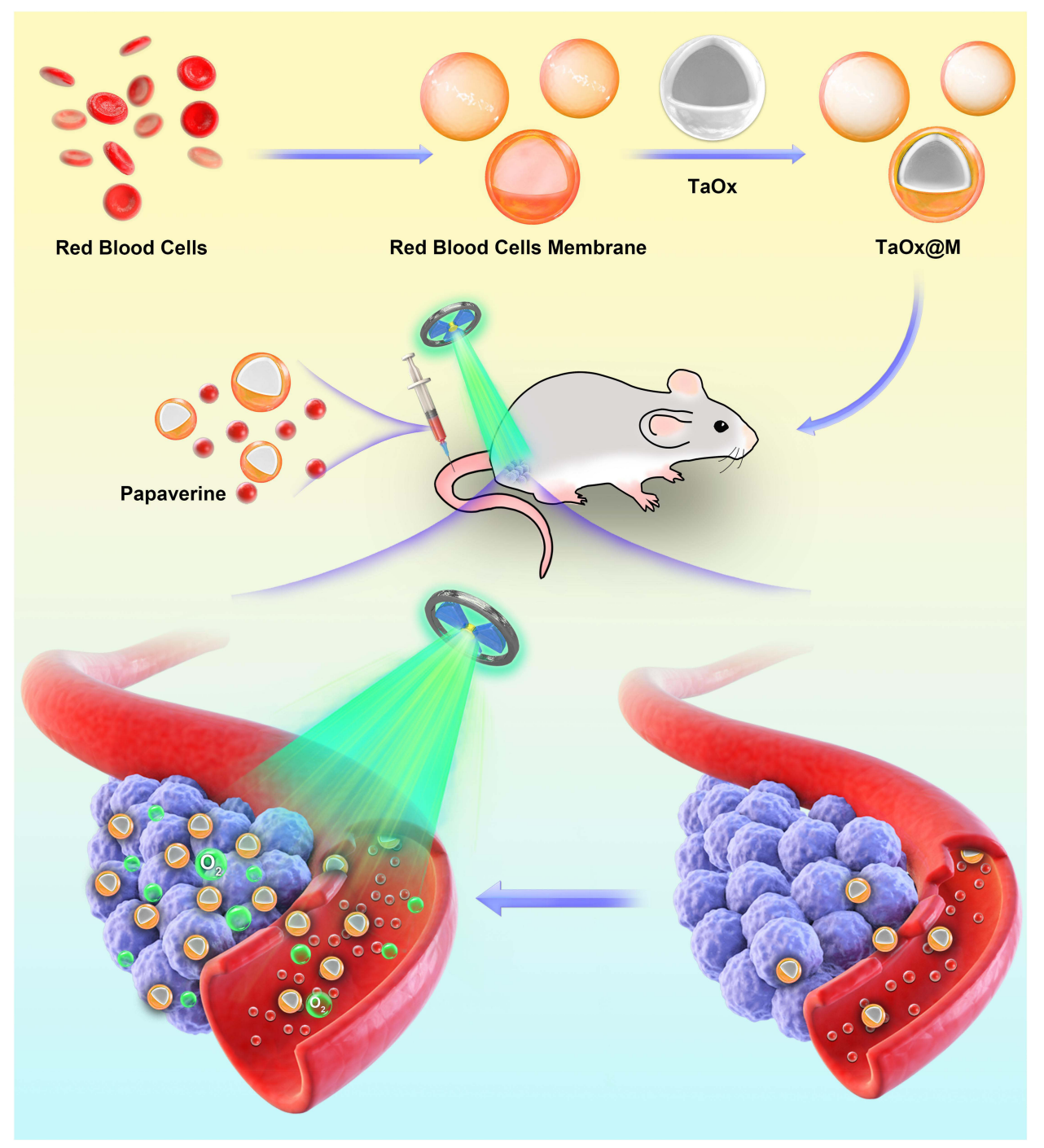

Scheme I Illustration of TaOx@M and ppv application. With vasomodulatory activity, paperverine improves the oxygen levels in cancerous tissue and reduces underperfused areas of the tumor, allowing for the thorough penetration and accumulation of TaOx@M.

$800 \times \mathrm{g}$ for $5 \mathrm{~min}$ at $4{ }^{\circ} \mathrm{C}$ to remove the plasma and the buffy coat. The resulting RBCs were washed three times with ice-cold $1 \times$ PBS. Then, $0.25 \times$ PBS $($ PBS: deionized water $=1: 3$ ) was added for hemolysis using a hypotonic medium treatment in an ice-bath for $30 \mathrm{~min}$. The released hemoglobin was removed by centrifugation at $1500 \mathrm{~g}$ for $5 \mathrm{~min}$, and the RBC-vesicles (light pink precipitate) were collected and washed twice with $1 \times$ PBS.

To encapsulate the $\mathrm{TaOx}, \mathrm{RBC}$-vesicles derived from $10 \mu \mathrm{L}$ of mouse blood were first mixed with $50 \mu \mathrm{g}$ of $\mathrm{TaOx}$ in $1 \mathrm{~mL}$ of PBS. Subsequently, the mixture was repeatedly extruded through a $200-\mathrm{nm}$ nucleopore polyester membrane on a mini-extruder and then centrifuged at $1000 \times g$ for $10 \mathrm{~min}$ to eliminate excess RBC-vesicles. The extruder was composed of two $10 \mathrm{~mL}$ syringes with needle removed. The two syringes were connected with the 200-nm nucleopore polyester membrane in the middle. Finally, the resulting RBC-TaOx were stored in PBS at $4{ }^{\circ} \mathrm{C}$ for the following experiments.

\section{Cell Cytotoxicity}

For the cell cytotoxicity assessment, K7M2 cells at the logarithmic growth phase were seeded into 96-well plates at a density of $5 \times 10^{3}$ cells per well in triplicate and incubated for $24 \mathrm{~h}$ at $37{ }^{\circ} \mathrm{C}$ with $\mathrm{CO}_{2}$ concentration of 5\%. Next, the cells were incubated with TaOx@M at various concentrations ranging from 25 to $200 \mu \mathrm{g} / \mathrm{mL}$, and cell viability was determined using a cell counting assay (CCK 8, KeyGEN). To study the therapeutic effects of various treatment methods, $5 \times 10^{3}$ cells were seeded 
into 96-well plates, with three replicants per group. The Control group was set up without any treatment, and the RT group was subjected to RT (5 Gy). For the experimental groups, cells were treated with $\mathrm{TaOx}(50 \mu \mathrm{g} / \mathrm{mL})$ or $\mathrm{TaOx} @ \mathrm{M}$ (quantity of TaOx@M equal to TaOx $50 \mu \mathrm{g} /$ $\mathrm{mL}$ ), and $12 \mathrm{~h}$ after the incubation, experimental cells received a radiation dose of 5 Gy. A standard CCK 8 assay was conducted to assess cell viability in each group.

\section{Clonogenic Assay}

The K7M2 cells were digested and seeded into 6-well plates at densities of 100, 200, 400, 800, and 1000 cells per well followed by incubation with TaOx or TaOx@M. After $12 \mathrm{~h}$, the cells were irradiated with 2, 4, 6, or 8 Gy $\mathrm{X}$-rays on a Small Animal Radiation Research Platform (PXI X-RAD 225Cx, CT, USA) with a 204-kV photon beam. After the residual nanoparticles were removed, the cells were cultured for 15 days then dyed with crystal violet. Each crystal violet dot was counted as a cell cluster. The survival curve was fitted using a "multitarget-singlehitting model".

\section{Animal Model}

The animals used in this study were obtained from the Vital River Company (Beijing, China). To establish tumorbearing models, $1 \times 10^{6}$ cells suspended in $100 \mu \mathrm{L}$ of PBS were subcutaneously injected into the right hip of each female Balb/c mouse (aged 6-8 weeks). All the animal experiments were performed according to the protocols of the Institutional Animal Care and Use Committee (IACUC) of the Animal Experiment Center of Wuhan University (Wuhan, China) with approval number of AF146. All experiments were performed in accordance with the Guidelines for Care and Use of Laboratory Animals of Wuhan University.

\section{Antitumor Efficacy of Combination Therapy}

When the tumor volume reached $200 \mathrm{~mm}^{3}$, the mice were randomly divided into six groups and subjected to the following treatments, hereafter referred to as day 0: (1) Control; (2) RT; (3) ppv + RT; (4) TaOx@M + RT; (5) $\mathrm{TaOx}+\mathrm{ppv}+\mathrm{RT}$; (6) TaOx@M + ppv + RT. The TaOx (TaOx dose: $50 \mathrm{mg} / \mathrm{kg}$ ) or TaOx@M (TaOx dose: $50 \mathrm{mg} /$ $\mathrm{kg}$ ) was injected into the mice via the tail vein. After $5 \mathrm{~h}$, the mice in groups 5 and 6 received $2 \mathrm{mg} / \mathrm{kg}$ body weight of ppv hydrochloride by intraperitoneal injection, $1 \mathrm{~h}$ after which the mice in groups 3,4 , and 5 were irradiated with $\mathrm{X}$-rays at a 5 Gy dose. It must be noted that ppv is unstable in saline and, thus, it was suspended in glucose carrier $(5 \%)$ in this study. The tumor growth inhibition (TGI) rate was calculated using the formula: $\mathrm{TGI}=1-\mathrm{V}_{\mathrm{t}} / \mathrm{V}_{\mathrm{c}}$, where $\mathrm{V}_{\mathrm{t}}$ and $\mathrm{V}_{\mathrm{c}}$ represent the average tumor volume in the treated group and control group, respectively. The combined TGI of a group a and group $b$ was determined using the formula: TGI $=1-\left(\mathrm{V}_{\mathrm{a}} \cdot \mathrm{V}_{\mathrm{b}}\right) / \mathrm{V}_{\mathrm{c}}^{2}$, where $\mathrm{V}_{\mathrm{a}}$ and $\mathrm{V}_{\mathrm{b}}$ represent the average tumor volume in the treated group and control group, respectively. The tumor growth and body weight were monitored every other day until the mice were sacrificed on day 15 . The tumors were surgically resected, and the tumors were weighed.

\section{Photoacoustic Imaging of Oxygenation}

The vascular blood saturation $\left(\mathrm{sO}_{2}\right)$ of the solid tumors was measured on a VevoLAZR system (Fujifilm, Visualsonics Inc. Canada). Female Balb/c mice with a tumor volume of approximately $200 \mathrm{~mm}^{3}$ were selected for in vivo imaging. For observation, the hair on the right hip of the mice was removed. After the mice were anesthetized with $2 \%$ isoflurane and fixed on the platform of the photoacoustic (PA) imaging system, a layer of ultrasonic coupling gel was applied to the skin for observation. The PA images of mice before and 30 $\mathrm{min}, 1 \mathrm{~h}, 6 \mathrm{~h}$ and $12 \mathrm{~h}$ after injection of ppv $(2 \mathrm{mg} / \mathrm{kg}$ body weight) and $\mathrm{sO}_{2}$ in the tumor sites were recorded.

\section{Immunohistochemical Analysis}

TaOx@M (TaOx dose: 50 mg/kg) suspended in PBS was intravenously injected into the K7M2-bearing mice before administration of ppv ( $2 \mathrm{mg} / \mathrm{kg}$ body weight) or saline through intraperitoneal injection $5 \mathrm{~h}$ later. Then, pimonidazole hydrochloride was intraperitoneally injected at the concentration of $60 \mathrm{mg}$ per $\mathrm{kg}$ of body weight. After 90 $\mathrm{min}$, the tumors were resected, frozen, and stained, and the images were visualized using confocal microscopy.

\section{Pharmacokinetics and Biodistribution}

We injected TaOx, TaOx@M, or TaOx@M + ppv into Balb/c mice via the tail vein in triplicate. For the $\mathrm{TaOx} @ \mathrm{M}+$ ppv group, the TaOx@M ( $\mathrm{TaOx}$ dose: $50 \mathrm{mg} / \mathrm{kg}$ ) was administered $5 \mathrm{~h}$ before injecting ppv ( $2 \mathrm{mg} / \mathrm{kg}$ body weight). Blood samples were collected from an eye socket using EDTA blood collection tubes at $30 \mathrm{~min}, 1 \mathrm{~h}, 2 \mathrm{~h}, 4 \mathrm{~h}, 8 \mathrm{~h}, 12 \mathrm{~h}$, and $24 \mathrm{~h}$ post-injection. After the samples were digested in concentrated $\mathrm{HNO}_{3}$, the concentration of tantalum in all tissues was measured 
using Inductively coupled plasma mass spectrometry (ICPMS). Pharmacokinetic parameters were calculated to fit with a two-compartmental model which is the most common model for describing the disposition of intravenously administered medicine using Graphpad Prism 8. The mice were euthanized at $24 \mathrm{~h}$ post-injection. The main organs, including the hearts, livers, spleens, lungs, and kidneys, as well as tumors were harvested and dissolved in concentrated $\mathrm{HNO}_{3}$. The tantalum content was analyzed using ICP-MS

\section{Hematological Evaluation and Histological Examination}

The 6 to 8-week old mice were administered TaOx@M ( $\mathrm{TaOx}$ dose: $50 \mathrm{mg} / \mathrm{kg})+$ ppv $(2 \mathrm{mg} / \mathrm{kg}$ body weight $)$. Blood samples were collected for analysis before the mice were dissected 30 days after injection. The main organs, including the heart, liver, spleen, lung, and kidney, were sectioned and stained with hematoxylin-eosin. An inverted laboratory microscope was used to capture images.

\section{Statistical Analysis}

Data are presented as mean \pm standard deviation (SD). Statistical differences were assessed using the student's t-test. P-values of $<0.05$ were considered statistically significant, and $*$ means $\mathrm{P}<0.05, * *$ means $\mathrm{P}<0.01$, and $* * *$ means $\mathrm{P}<0.005$.

\section{Results and Discussion}

\section{Synthesis and Characterization of TaOx@M}

To prepare the TaOx@M, hollow TaOx was first synthesized via the one-pot method by the simple addition of tantalum (V) ethoxide to a mixture of water and ethanol at room temperature, as previously reported..$^{19}$ Next, the TaOx was coated with RBC membranes using an extrusion method. The transmission electron microscopy (TEM) images (Figure 1A and B) show the uniform hollow structure of the TaOx and the core-shell structure of TaOx@M with a thin lipid bilayer. Further investigation using high-angle annular dark-field scanning TEM (HAADF-STEM) confirmed the presence of a $\mathrm{TaOx}$ core with a diameter of 85.6 $\mathrm{nm}$ and a 6.8-nm-thick membrane shell (Figure 1C), indicating a lipid bilayer thickness of approximately $7 \mathrm{~nm}$, which is consistent with the RBC membrane width. Additionally, the measurement of dynamic light scattering gave an average diameter of $97.1 \mathrm{~nm}$ and $106.2 \mathrm{~nm}$ for TaOx and TaOx@M, respectively (Figure 1D). In addition, zeta-potential measurements (Figure 1E) exhibited a shift in the zeta-potential from -20.1 mV (TaOx) to -26.8 mV (TaOx@M), which are close to the zeta-potential of $\mathrm{RBC}$ vesicles $(-33.2 \mathrm{mV})$. Further SDS-PAGE analysis whose experiment detail was shown in supporting information 1.3 verified the maintenance of most cell membrane components in TaOx@M (Figure 1F), indicating that membrane-associated protein functions were preserved. The above results demonstrated that $\mathrm{TaOx}$ was successfully cloaked with the RBC membrane. In contrast to $\mathrm{TaOx}$, which showed low stability in physiological solutions, TaOx@M exhibited superior stability in both saline and serum for up to $24 \mathrm{~h}$ (Figure S1 as shown in the supporting information file). All the instruments were shown in supporting information 1.2.

\section{Radiosensitization in vitro}

First, the hematolysis rate of TaOx@M was less than 5\%, even at a concentration of $200 \mu \mathrm{g} / \mathrm{mL}$, indicating there was no obvious risk of damage to the erythrocytes (Figure S2 as shown in the supporting information file). We evaluated the biocompatibility of TaOx and TaOx@M using a cell counting kit (CCK) 8 assay following the cell culture method in supporting information 1.4. As shown in Figure S3 as shown in the supporting information file, both TaOx and TaOx@M exhibited minimal cytotoxicity to K7M2, even at high concentrations, suggesting the biomedical application of TaOx and TaOx@M is feasible. $\mathrm{TaOx}$ can act as a radiosensitizer because of its high atomic number, according to a previously reported study. ${ }^{19}$ Next, we compared the radiosensitizing effect of TaOx@M under both hypoxic and normoxic conditions. $\gamma$ $\mathrm{H}_{2} \mathrm{AX}$ is a marker of DNA double-strand breaks. As shown in Figure 2A and B, cells pretreated with TaOx@M exhibited higher levels of DNA damage than those treated with RT alone in normoxic conditions. However, because oxygen is indispensable in preventing the repair of DNA radicals, the DNA damage was highly suppressed under hypoxic conditions, whether the cells were incubated with TaOx@M or not. Cells treated with TaOx@M prior to irradiation with 5 Gy showed a decrease in cell viability with an increase in the concentration of TaOx@M under normoxia; in contrast, cell viability was consistently high in hypoxic conditions at all concentrations (Figure 2C). The above results demonstrated that TaOx@M possesses remarkable radiosensitization ability in normoxia but this ability was limited in hypoxia situation, which is common in solid tumors. A clonogenic assay was used as a "gold standard" to evaluate the 
A

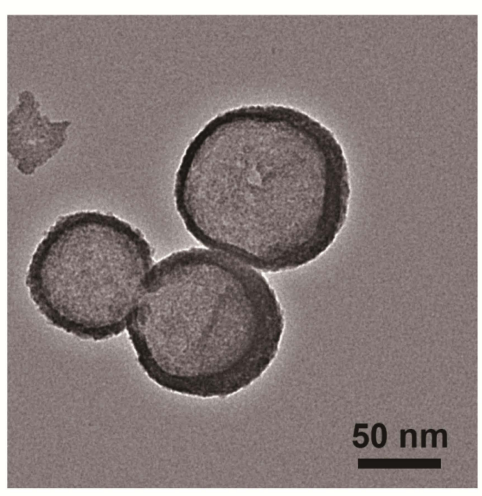

D

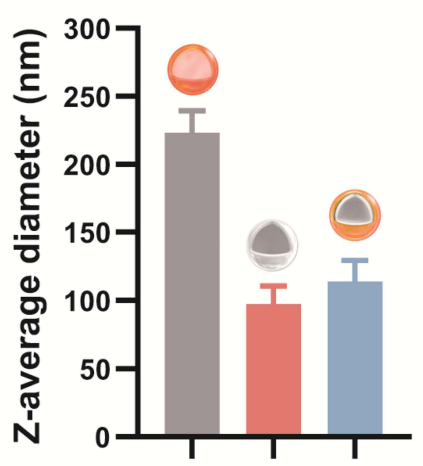

B

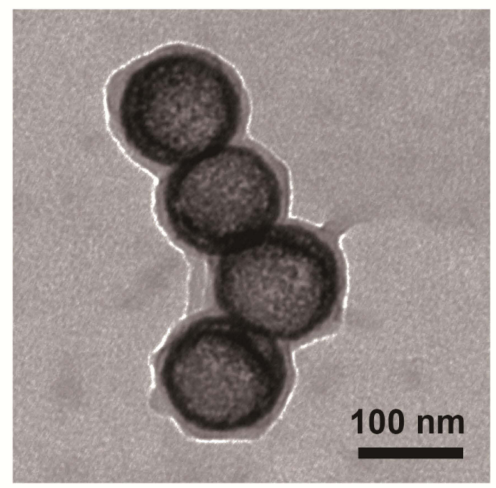

E

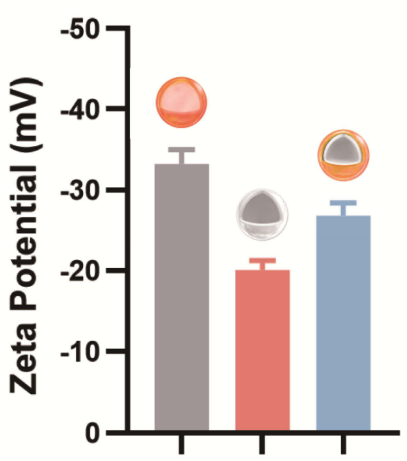

C
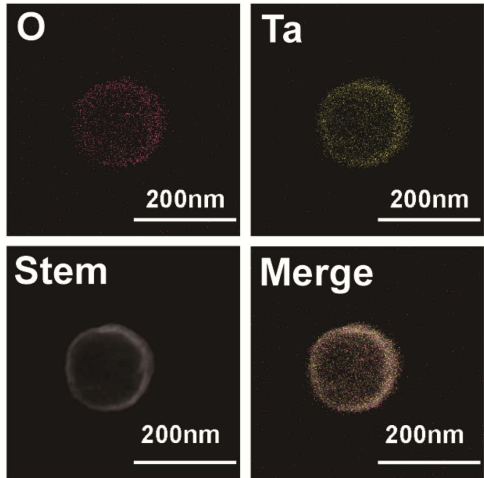

Merge

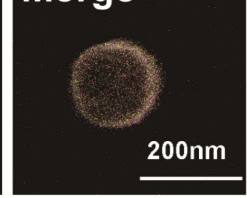

$\mathbf{F}$

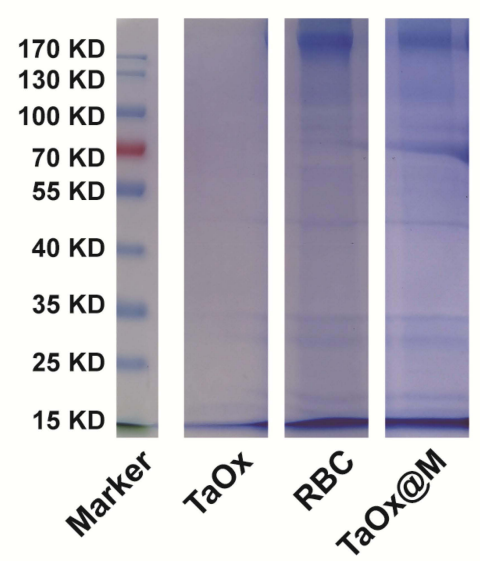

Figure I Characterization of TaOx@M nanoparticles. (A, B) TEM images of TaOx and TaOx@M, respectively. (C) HAADF-STEM image and elemental mapping of TaOx. (D) Size distribution of RBC membrane, TaOx, and TaOx@M. (E) Surface zeta-potentials of TaOx and TaOx@M, respectively. (F) Proteins in RBC membrane; TaOx, and TaOx@M were solubilized and resolved on a polyacrylamide gel.

proliferation ability of cancerous cells (Figure 2D). Under hypoxic conditions, both RT and TaOx@M + RT exhibited a limited inhibitory effect on cell proliferation. In comparison, there was an obvious separation between the TaOx@M + RT curve under normoxic conditions and that under hypoxic conditions, which highlights the importance of oxygen during RT. The sensitizing enhancement ratio, which is representative of enhancement efficiency, was calculated to be 1.93 for TaOx@M under normoxia, indicating that the $\mathrm{RBC}$-mimicking $\mathrm{TaOx}$ effectively radiosensitized $\mathrm{K} 7 \mathrm{M} 2$ cells.

\section{Oxygenation and Vasodilation by Ppv}

In the majority of cancers, the blood vessels have abnormal structure and function, which fuel disease progression and resistance to treatment. ${ }^{50}$ Therefore, it is advantageous to optimize the blood flow in tumors. Considering the potent vasodilatory effect of ppv accelerates blood flow, the intermittent blood flow induced by the abnormal vasculature hinders the delivery of oxygen to tumorous tissues. Because the velocity of the blood flow is proportional to vessel diameter, the blood flow and oxygen status may be altered after the injection of ppv. ${ }^{51}$ To validate the hypoxia-alleviating capability of ppv, in vivo PA imaging was performed on living mice bearing K7M2 tumors to observe the vascular blood saturation $\left(\mathrm{sO}_{2}\right)$ in real-time. As illustrated in Figure 3A, PA signals at the tumor sites gradually increased and reached the maximum at $1 \mathrm{~h}$ post-injection, with a signal intensity 5.3 -fold that before injection and the PA signal sustained at a relative high intensity at $12 \mathrm{~h}$ post the injection (Figure 3B). Because ppv improved the vascular blood flow and, 


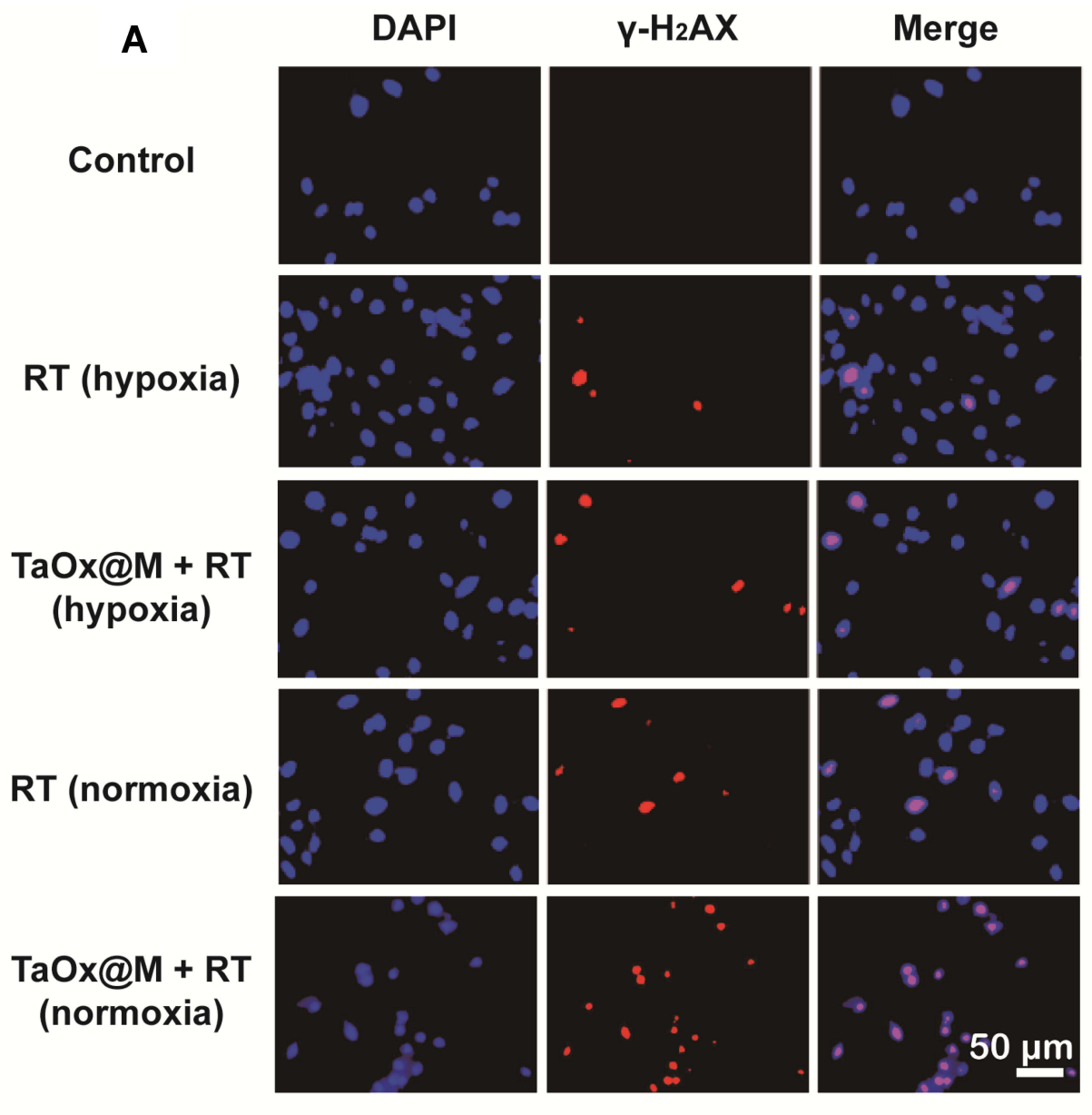

B

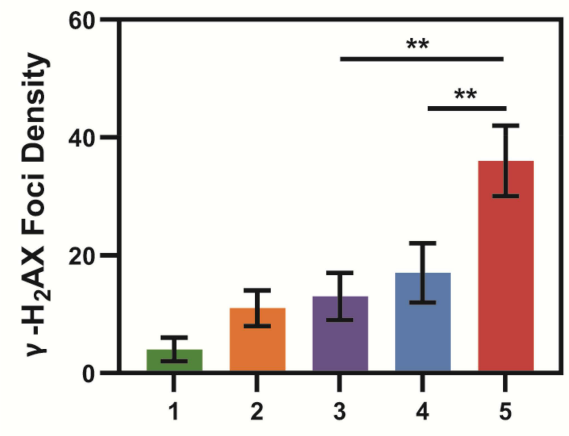

C

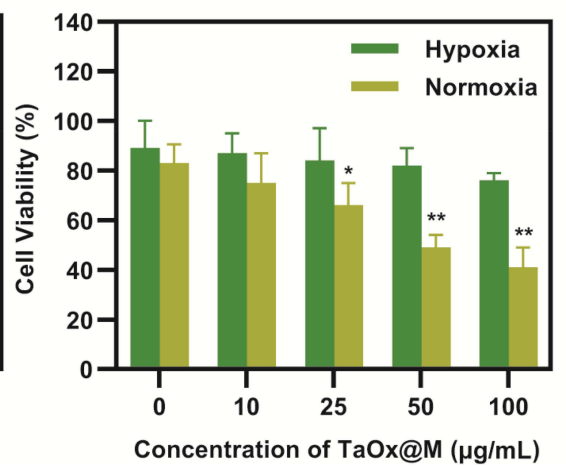

D

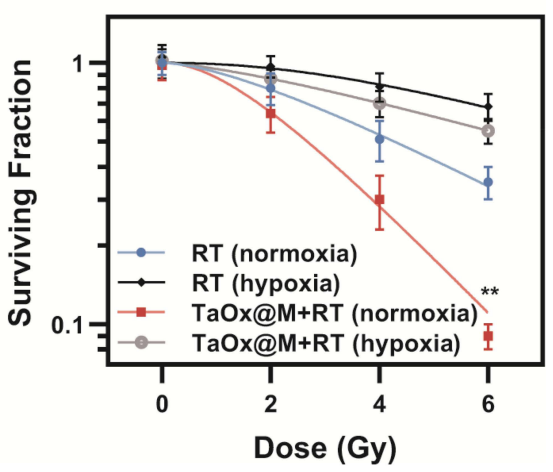

Figure 2 In vitro radiosensitization with TaOx@M. (A) Confocal fluorescence image of $\gamma-\mathrm{H}_{2} \mathrm{AX}$-stained K7M2 cells treated with PBS or TaOx@M (50 $\mu$ g/mL) with or without radiation (5 Gy). (B) Quantitative analysis of $\gamma-\mathrm{H}_{2} \mathrm{AX}$ foci density $\left(\gamma-\mathrm{H}_{2} \mathrm{AX}\right.$ foci/l $\left.00 \mu \mathrm{m}^{2}\right)$ for $\mathrm{n}>\mathrm{I} 00$ cells. Group I: PBS; Group 2: RT (hypoxia); Group 3: TaOx@M + RT (hypoxia); Group 4: RT (normoxia); Group 5: TaOx@M + RT (normoxia). (C) Cell viabilities of K7M2 cells incubated with TaOx@M at various concentrations under hypoxia or normoxia. (D) Clonogenic curves. The K7M2 cells were treated with PBS or TaOx@M (50 $\mu$ g/mL) and irradiated with X-rays of various dosages. All data are presented as mean \pm standard deviation (SD). ${ }^{*} \mathrm{p}<0.05$, ${ }^{*} \mathrm{p} p<0.0 \mathrm{I}$, and $*^{* * *} \mathrm{p}<0.00 \mathrm{I}$.

therefore, increased the intratumor blood oxygen saturation, it was highly beneficial in relieving hypoxia. To further verify its hypoxia alleviation effectiveness, tumors of mice that were concurrently treated with ppv were sectioned for HIF-1 $\alpha$ immunofluorescent (green fluorescent) staining. As shown in Figure 3C, the expression levels of the HIF- $1 \alpha$ protein in tumors significantly decreased over time after the injection of ppv, demonstrating the potent ability of ppv to relieve hypoxia. The intensity of HIF-1 $\alpha$ fluorescence at 30 min post-injection 
A
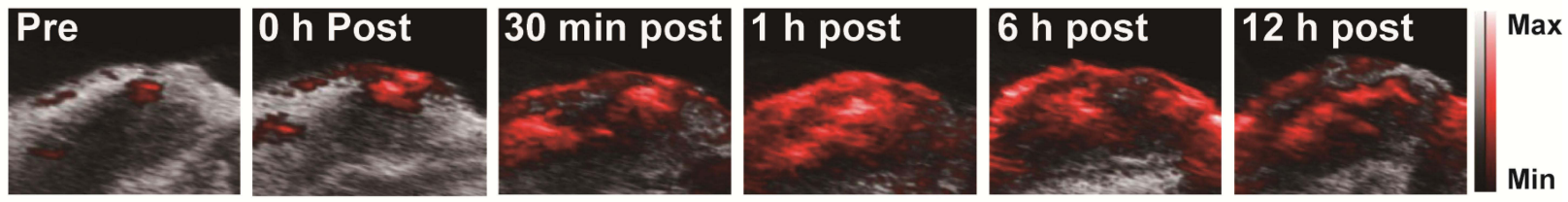

B

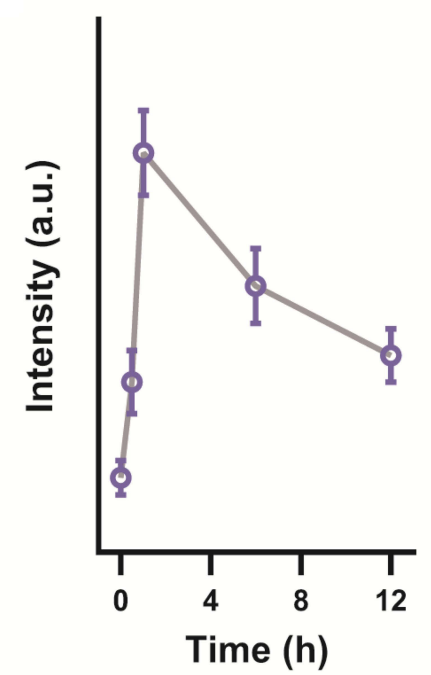

C

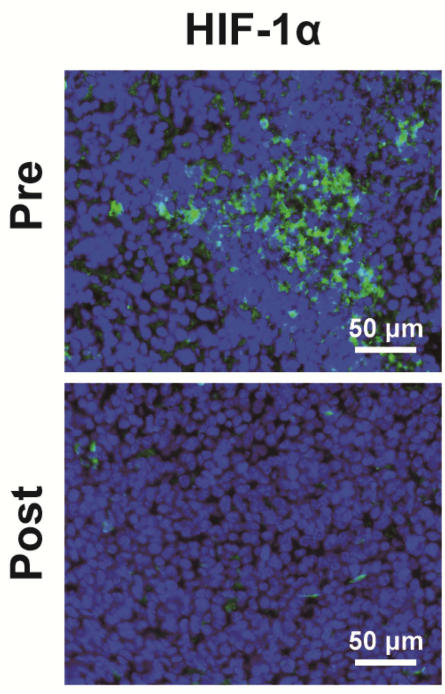

D

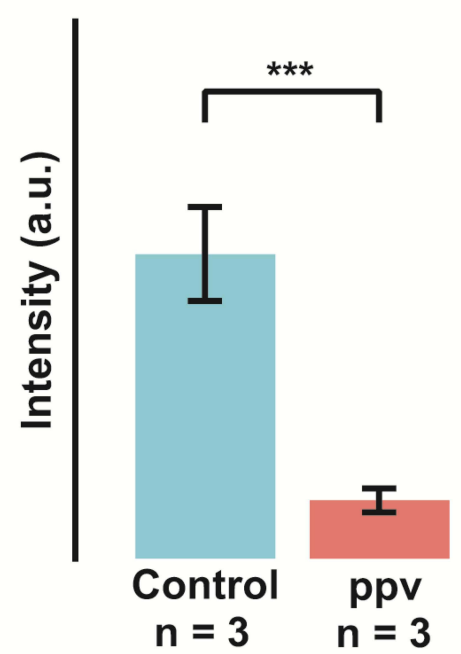

Figure 3 Hypoxia alleviation. (A) The $\mathrm{sO}_{2}$ content and (B) intensity of PA signals before and $0 \mathrm{~h}, 30 \mathrm{~min}, \mathrm{I}$ h, $6 \mathrm{~h}$ and $12 \mathrm{~h}$ after injection of ppv. (C) Micrographs of tumor slices stained with HIF-I $\alpha$ (green) and (D) corresponding quantified fluorescence intensity. All data are presented as mean \pm standard deviation (SD). *p $<0.05$, **p $<0.0 \mathrm{I}$, and $* * * p<0.001$.

was 5.2-fold that before injection (Figure 3D), which was due to the elevated oxygen content in the tumorous region. Further investigation on hypoxia status of $0 \mathrm{~h}, 0.5 \mathrm{~h}$ and 1 $h$ post the injection of ppv was shown in Figure S4. It can be inferred that $1 \mathrm{~h}$ post the injection the hypoxia status was alleviated to the largest extent among these three time points. All the results so far suggest that ppv alleviated tumor hypoxia by accelerating blood flow.

\section{Ppv Facilitates TaOx@M Accumulation and Tumor Penetration}

Following our demonstration of the promising effects of ppv in vivo, we further investigated whether ppv has an impact on preferential TaOx@M accumulation and penetration at a systemic level. The K7M2 tumor-bearing mice were i.v. injected with $\mathrm{TaOx}$ (Group 1), $\mathrm{TaOx} @ \mathrm{M}$ (Group 2), or ppv + TaOx@M (Group 3). Following animal sacrifice, we studied the pharmacokinetic profiles of the three groups. Both Group 2 and 3 showed a prolonged blood circulation half-time relative to Group 1, which was attributed to the cloaking of $\mathrm{TaOx}$ by the $\mathrm{RBC}$ membrane (Figure 4A). The distribution of tantalum in the major organs and tumors was measured (Figure 4B) and, for all groups, the majority of nanoparticles were in the liver and spleen - two primary organs of the RES. Tantalum contents were lower in the liver and spleen and higher in the tumors of mice treated with TaOx@M compared with those treated with $\mathrm{TaOx}$, because the erythrocyte coat was "invisible" to the immune system. However, Group 3 showed the highest accumulation of tantalum in tumorous tissues as well as the lowest tantalum contents in the liver and spleen compared with the other groups. The above results suggest that ppv enables $\mathrm{TaOx}$ accumulation within tumors and prevents its accumulation in the liver and spleen. Therefore, nanoparticles are cleared more slowly from tumor tissues, increasing the efficacy of the RT. Next, to compare the effect of ppv on biodistribution, dye-labeled TaOx@M were injected via tail vein and ex vivo imaging was conducted to confirm the biodistribution. In ppv+TaOx@M group, ppv was administrated before TaOx@M. As shown in Figure 4C and D, ppv+TaOx@M group exhibited higher signal intensity than that of TaOx@M group, which was consistent with the result in vivo. Moreover, we monitored the tissue accumulation at tissue level. As shown in Figure 4E, more DiO- 
A

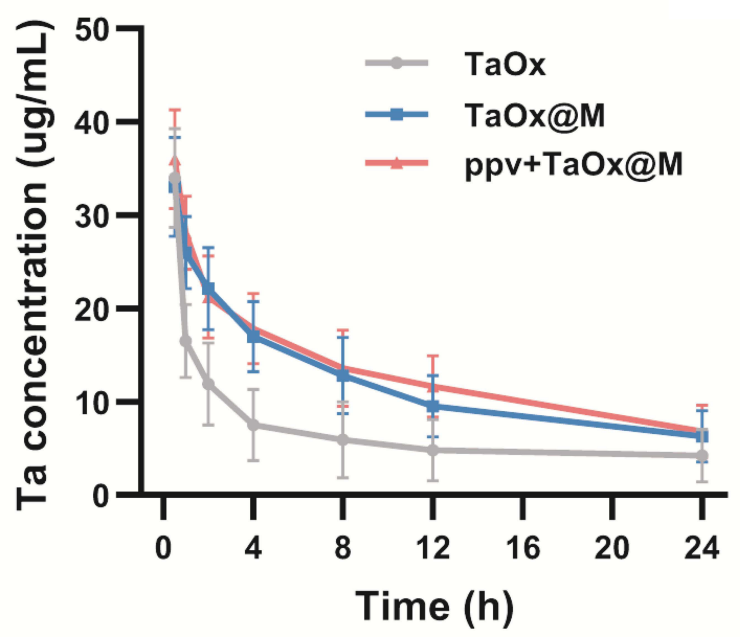

\section{TaOx@M}
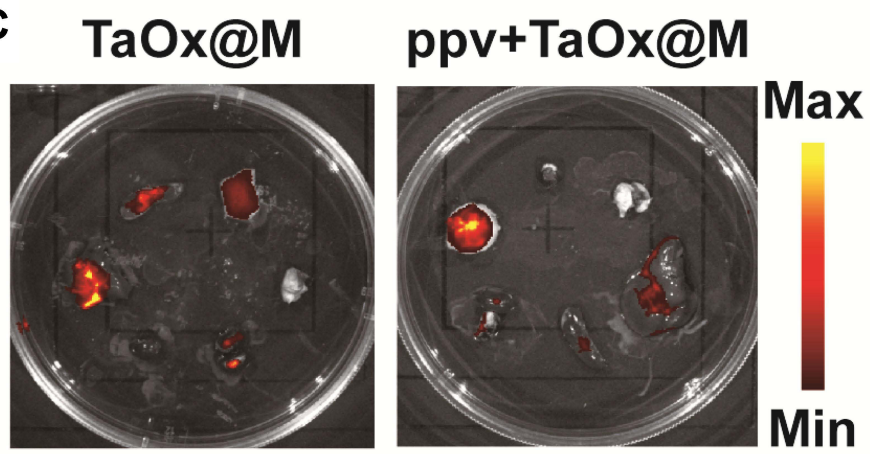

E

TaOx@M

B

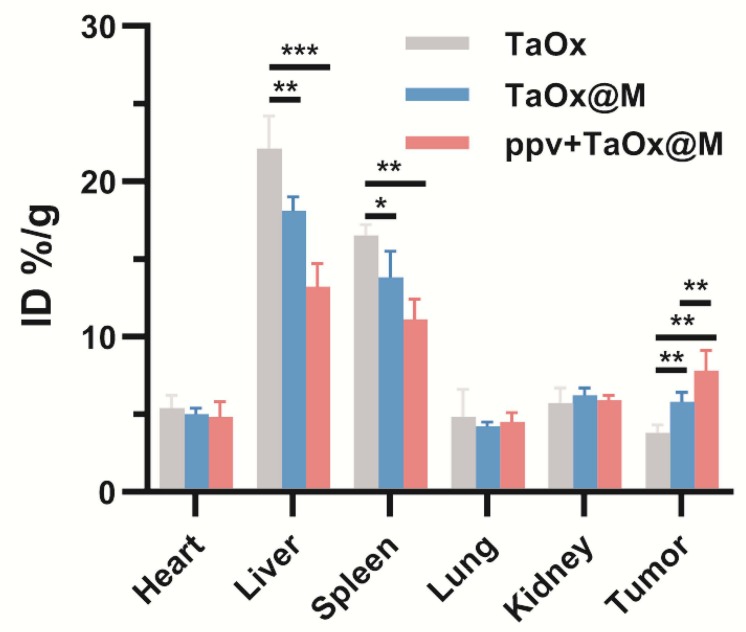

D

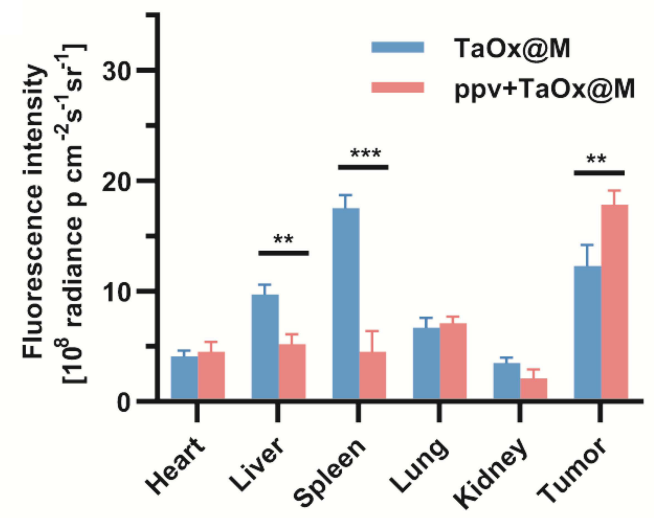

$\mathbf{F}$

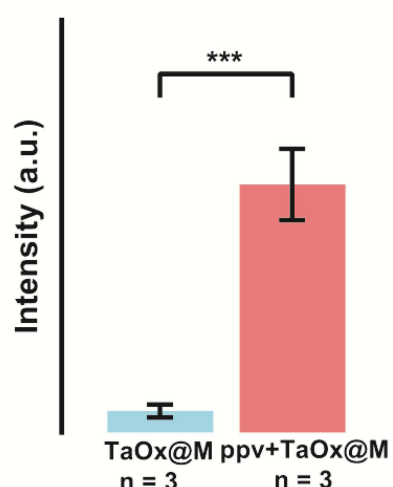

Figure 4 Enhanced penetration and accumulation of ppv. (A) Pharmacokinetic study of the various formulations. (B) Biodistribution of tantalum in tumors and major organs after various treatments. (C) Ex vivo fluorescence imaging and (D) quantitative data of fluorescence signals in main organs and tumors. (E) Immunofluorescence staining imaging of the intratumoral distribution of DiO-labeled TaOx@M, cell nuclei was stained with 2-(4-amidinophenyl)-IH-indole-6-carboxamidine (blue) and TaOx@M was labeled with $\mathrm{DiO}$ (green) and (F) quantitative analysis of fluorescence signals of Dio-labeled TaOx@M. All data are presented as mean \pm standard deviation (SD). *p < 0.05, $*^{*} \mathrm{p}<0.01$, and $* * *_{\mathrm{p}}<0.001$.

labeled TaOx@M were found in ppv+TaOx@M group however lower green fluorescence signals were observed in $\mathrm{TaOx} @ \mathrm{M}$, which was identified with the quantitative results of fluorescence intensity in Figure 4F. It should be notified that the ppv is capable of improving the penetration ability of nanoparticles. These results clearly verified the importance of ppv on enhancing accumulation of TaOx@M in tumor tissues. 


\section{Biosafety Assessment}

Before studying the antitumor treatments in vivo, their biosafety was assessed in Balb/c mice. In order to investigate the risk of ppv and TaOx@M may pose to healthy tissues in a relative long term, we assessed the hematological parameters of mice 30 days after treatment with TaOx@M and ppv. As shown in Figure 5A, there were negligible differences among the groups. Moreover, histological examination revealed no apparent lesions or apoptosis in the main organs in any of the groups (Figure 5B). Collectively, ppv and $\mathrm{TaOx} @ \mathrm{M}$ showed no significant toxicity on the organs.

\section{Antitumor Efficacy}

To verify the effectiveness of using ppv prior to TaOx@M for radiosensitization, mice were divided randomly into five groups: Group 1: saline control; Group 2: RT only; Group 3: ppv + RT; Group 4: TaOx@M + RT; Group 5: ppv + TaOx + RT; and Group 6: ppv + TaOx@M + RT. The mice were treated with or without ppv after the administration of $\mathrm{TaOx}$ or $\mathrm{TaOx} @ \mathrm{M}$, followed by X-ray irradiation at 5 $\mathrm{h}$ post-injection. The treatment procedure for Group 6 is provided as an example in Figure 6A. There was no obvious difference in body weights among the groups (Figure 6B). As shown in Figure 6C, RT alone did not effectively suppress the proliferation of tumors, whereas a slightly better tumor inhibition of 54.3\% was achieved when TaOx@M was introduced as a radiosensitizer. Consistent with a previously reported study, which showed that ppv and its derivatives enhanced the response of solid tumors to RT, $\mathrm{ppv}+\mathrm{RT}$ showed $46.6 \%$ TGI. Moreover, the $\mathrm{ppv}+\mathrm{TaOx}+$

\section{A}
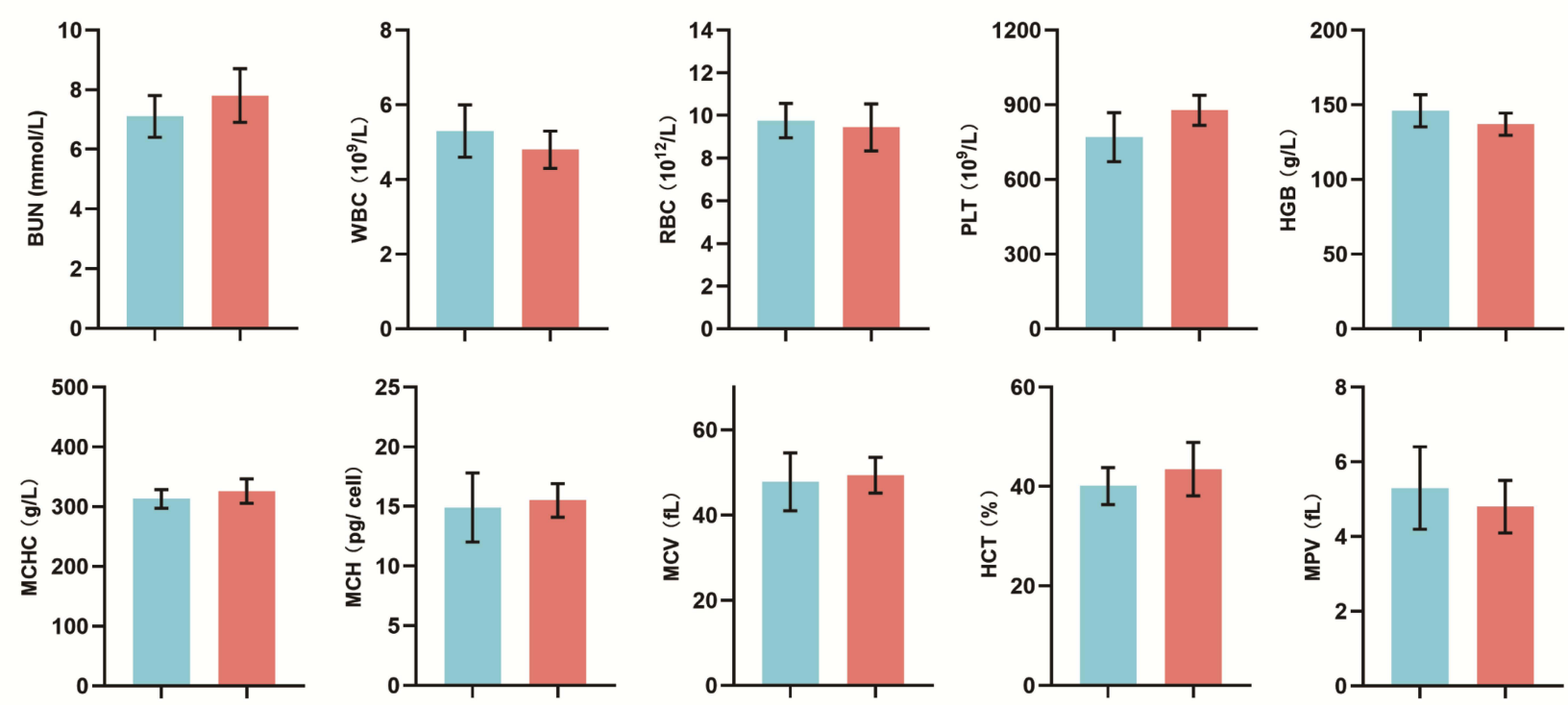

B
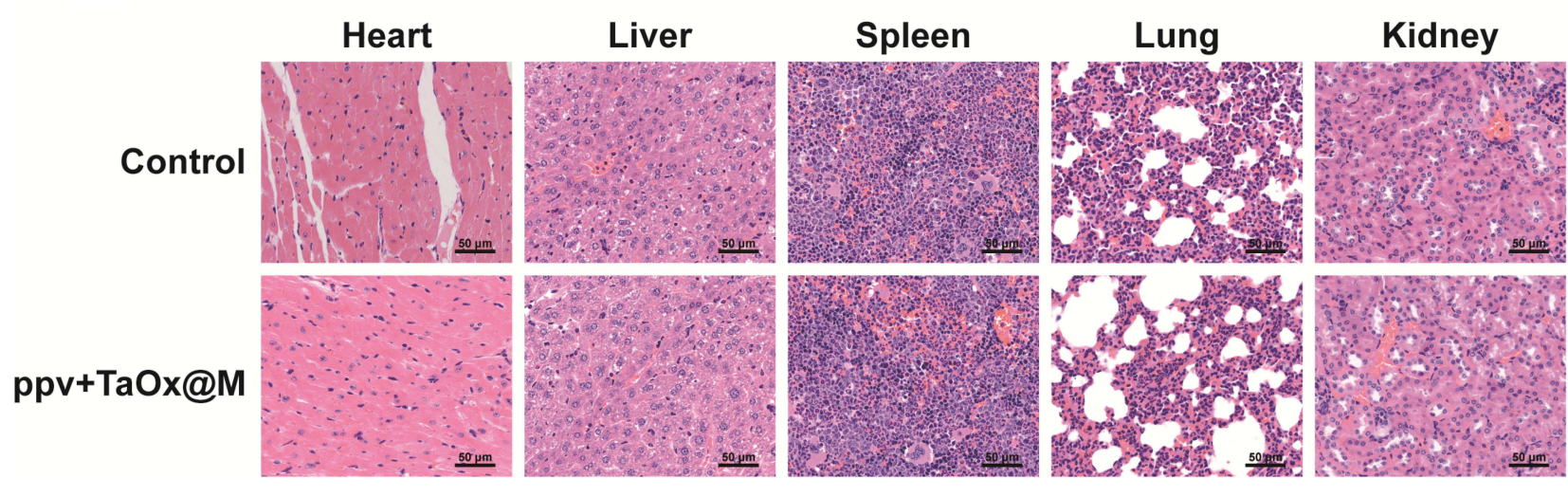

Figure 5 In vivo toxicity evaluations. (A) Blood biochemistry analysis after various treatments. Blue: control group; red: ppv + TaOx@M group. (B) Micrographs of hematoxylin and eosin staining of main organs after various treatments. 
A

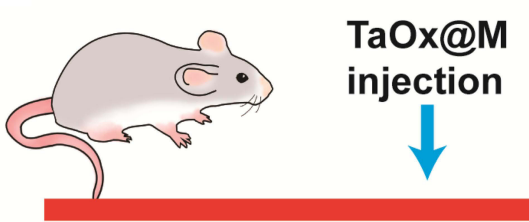

0
C

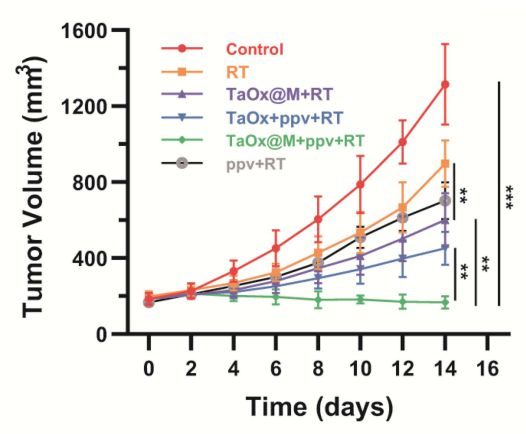

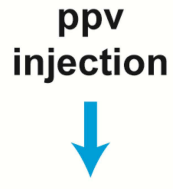

$5 \mathrm{~h}$

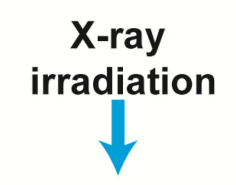

B

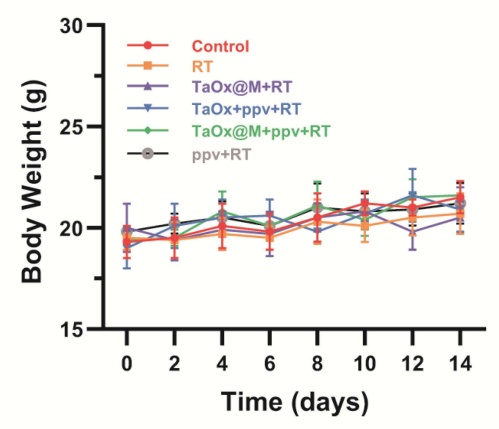

E
Control

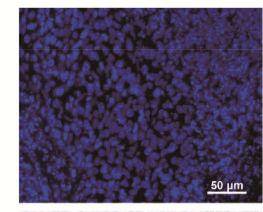

H\&E

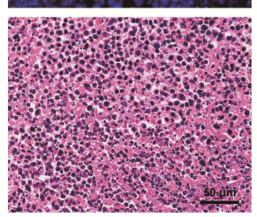

\section{RT}
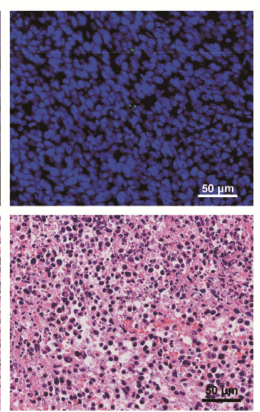

TaOx@M

ppv+RT
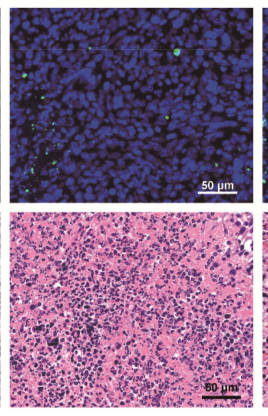

$+\mathrm{RT}$
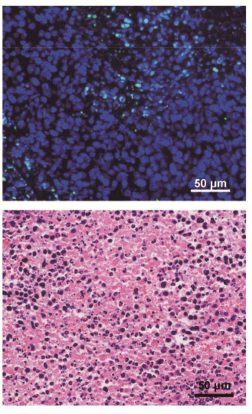

$6 \mathrm{~h}$

D

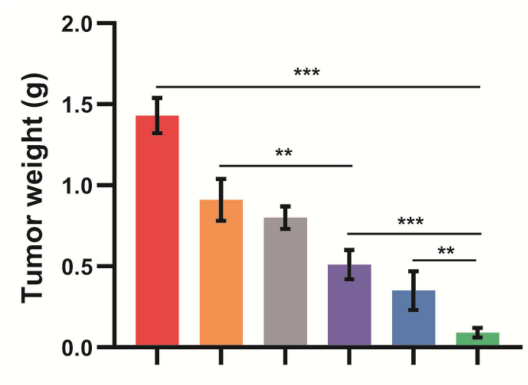

Figure 6 In vivo therapy evaluation. (A) Illustration of therapy procedure. Changes in (B) body weight, (C) tumor volume, and (D) tumor weight of mice in various groups. Red: control group; Orange: RT group; gray: ppv + RT; purple: TaOx@M + RT; blue: PPv + TaOx + RT; green: PPv + TaOx@M + RT. (E) Histological TUNEL and H\&E staining of sections of tumor slices after various treatments. All data are presented as mean \pm standard deviation (SD). ${ }^{*} p<0.05$, ** $p<0.0 \mathrm{I}$, and $* * * p<0.00 \mathrm{I}$.

RT group exhibited a high TGI ratio of $65.7 \%$. Notably, ppv+TaOx@M + RT showed the most remarkable tumor growth inhibition, with a dramatic increase in the TGI ratio (87.3\%), a 1.61-fold increase from that of Group 4; hence, the administration of $\mathrm{ppv}$ after the radiosensitizer led to significantly enhanced RT. In addition, the TGI in Group 6 was higher than the TGIs $(75.6 \%)$ of groups 3 and 4 combined, indicating that the therapy had a synergistic effect. Moreover, we observed a stronger antitumor effect from ppv+TaOx@M + RT compared with ppv + TaOx + RT as a result of the camouflage effect of the erythrocyte membrane. Analysis of the tumor weights (Figure 6D) further confirmed that ppv + TaOx@M + RT generated the most effective tumor inhibition effect of all the treatments. Compared with the other treatments, which exhibited constrained tumor suppression, ppv + TaOx@M + RT resulted in severe structural disruption as well as the highest level of tumor apoptosis according to the histological analysis and TUNEL staining (Figure 6E).

\section{Conclusion}

We showed the feasibility of applying ppv injections to enhance the radiosensitization effect of TaOx@M. The proposed method provide possibility of improve the hypoxia status in tumor regions. The function of $\mathrm{ppv}$ was to induce vasodilation, which accelerated blood 
flow to improve oxygen delivery to the tumorous area, enabling the RT-induced suppression of DNA repair. Furthermore, in the in vivo experiments, ppv notably enhanced TaOx@M accumulation and penetration in tumors. Additionally, the RBC membrane coating camouflaged the nanoparticle, prolonging its circulation time and improving its retention by preventing RES clearance. A significant tumor inhibition effect $(87.3 \%)$ was observed when ppv and TaOx@M were used in combination as a radiosensitization strategy. The strategy presented here may provide a new method of overcoming impediments to the efficacy of RT in combating tumors.

\section{Supporting Information}

Supporting information is available from online or from the author.

\section{Acknowledgment}

We greatly acknowledge the financial support from the National Natural Science Foundation of China (No. 81901882). The authors would like to thank Shiyanjia lab (www.shiyanjia.com) for drawing schematic illustration.

\section{Disclosure}

The authors report no conflicts of interest in this work.

\section{References}

1. DeLaney TF, Park L, Goldberg SI, et al. Radiotherapy for local control of osteosarcoma. Int $J$ Radiation Oncol Biol Phys. 2005;61 (2):492-498. doi:10.1016/j.ijrobp.2004.05.051

2. Du C, Zhou M, Jia F, et al. D-arginine-loaded metal-organic frameworks nanoparticles sensitize osteosarcoma to radiotherapy. Biomaterials. 2021;269:120642. doi:10.1016/j.biomaterials.2020.120642

3. Chung AS, Lee J, Ferrara N. Targeting the tumour vasculature: insights from physiological angiogenesis. Nat Rev Cancer. 2010;10 (7):505-514. doi: $10.1038 / \mathrm{nrc} 2868$

4. Brown AG. The unique physiology of solid tumors: opportunities (and problems) for cancer therapy. Cancer Res. 1998;58(7):1408-1416.

5. Dewhirst MW, Cao Y, Moeller B. Cycling hypoxia and free radicals regulate angiogenesis and radiotherapy response. Nat Rev Cancer. 2008;8(6):425-437. doi:10.1038/nrc2397

6. Song G, Cheng L, Chao Y, Yang K, Liu Z. Emerging nanotechnology and advanced materials for cancer radiation therapy. Adv Mater. 2017;29(32):1700996. doi:10.1002/adma.201700996

7. Liang S, Deng X, Chang Y, et al. Intelligent hollow pt-cus janus architecture for synergistic catalysis-enhanced sonodynamic and photothermal cancer therapy. Nano Lett. 2019;19(6):4134-4145. doi:10.1021/acs.nanolett.9b01595

8. Wang $\mathrm{D}, \mathrm{Wu} \mathrm{H}, \mathrm{Lim} \mathrm{WQ}$, et al. A mesoporous nanoenzyme derived from metal-organic frameworks with endogenous oxygen generation to alleviate tumor hypoxia for significantly enhanced photodynamic therapy. Adv Mater. 2019;31(27):e1901893. doi:10.1002/adma.2019 01893
9. Song X, Feng L, Liang C, Yang K, Liu Z. Ultrasound triggered tumor oxygenation with oxygen-shuttle nanoperfluorocarbon to overcome hypoxia-associated resistance in cancer therapies. Nano Lett. 2016;16(10):6145-6153. doi:10.1021/acs.nanolett.6b 02365

10. Yi X, Xu M, Zhou H, et al. Ultrasmall hyperbranched semiconducting polymer nanoparticles with different radioisotopes labeling for cancer theranostics. ACS Nano. 2018;12(9):9142-9151. doi:10.1021/ acsnano. $8 \mathrm{~b} 03514$

11. Zheng N, Wang Q, Li C, et al. Responsive Degradable theranostic agents enable controlled selenium delivery to enhance photothermal radiotherapy and reduce side effects. Adv Healthc Mater. 2021;10 (10):e2002024. doi:10.1002/adhm.202002024

12. Liu Y, Zhang P, Li F, et al. Metal-based Nanoenhancers for future radiotherapy: radiosensitizing and synergistic effects on tumor cells. Theranostics. 2018;8(7):1824-1849. doi:10.7150/thno.22172

13. Tang W, Dong Z, Zhang R, et al. Multifunctional TwoDimensional Core-Shell MXene@Gold Nanocomposites for Enhanced Photo-Radio Combined Therapy in the Second Biological Window. ACS Nano. 2018;13(1):284-294. doi:10.10 21/acsnano.8b05982

14. Cheng K, Sano M, Jenkins CH, et al. Synergistically Enhancing the Therapeutic Effect of Radiation Therapy with Radiation Activatable and Reactive Oxygen Species-Releasing Nanostructures. ACS Nano. 2018;12(5):4946-4958. doi:10.1021/acsnano.8b02038

15. Cheng X, Yong Y, Dai Y, et al. Enhanced Radiotherapy using Bismuth Sulfide Nanoagents Combined with Photo-thermal Treatment. Theranostics. 2017;7(17):4087-4098. doi:10.7150/thno. 20548

16. Deng J, Xu S, Hu W, Xun X, Zheng L, Su M. Tumor targeted, stealthy and degradable bismuth nanoparticles for enhanced X-ray radiation therapy of breast cancer. Biomaterials. 2018;154:24-33. doi:10.1016/j.biomaterials.2017.10.048

17. Li Y, Yun KH, Lee H, Goh SH, Suh YG, Choi Y. Porous platinum nanoparticles as a high- $Z$ and oxygen generating nanozyme for enhanced radiotherapy in vivo. Biomaterials. 2019;197:12-19. doi:10.1016/j.biomaterials.2019.01.004

18. Song G, Ji C, Liang C, et al. TaOx decorated perfluorocarbon nanodroplets as oxygen reservoirs to overcome tumor hypoxia and enhance cancer radiotherapy. Biomaterials. 2017;112:257-263. doi:10.1016/j.biomaterials.2016.10.020

19. Song G, Chao Y, Chen Y, et al. All-in-One Theranostic Nanoplatform Based on Hollow TaOx for Chelator-Free Labeling Imaging, Drug Delivery, and Synergistically Enhanced Radiotherapy. Adv Funct Mater. 2016;26(45):8243-8254. doi:10.1002/adfm.201603845

20. Rodriguez PL, Harada T, Christian DA, Pantano DA, Tsai RK, Discher DE. Minimal "Self" peptides that inhibit phagocytic clearance and enhance delivery of nanoparticles. Science. 2013;339 (6122):971-975. doi:10.1126/science.1229568

21. Minchinton AI, Tannock IF. Drug penetration in solid tumours. Nat Rev Cancer. 2006;6(8):583-592. doi:10.1038/nrc1893

22. Frank Alexis EP, Linda K, Omid C. Factors Affecting the Clearance and Biodistribution of Polymeric Nanoparticles. Mol Pharm. 2008;5 (4):505-515. doi:10.1021/mp800051m

23. Barua S, Mitragotri S. Challenges associated with Penetration of Nanoparticles across Cell and Tissue Barriers: a Review of Current Status and Future Prospects. Nano Today. 2014;9(2):223-243. doi:10.1016/j.nantod.2014.04.008

24. Zhang YR, Lin R, Li HJ, He WL, Du JZ, Wang J. Strategies to improve tumor penetration of nanomedicines through nanoparticle design. Wiley Interdiscip Rev Nanomed Nanobiotechnol. 2019;11 (1):e1519. doi:10.1002/wnan.1519

25. Li HJ, Du JZ, Du XJ, et al. Stimuli-responsive clustered nanoparticles for improved tumor penetration and therapeutic efficacy. Proc Natl Acad Sci U S A. 2016;113(15):4164-4169. doi:10.1073/pnas.15 22080113 
26. Lin X, Liu S, Zhang X, et al. An Ultrasound Activated Vesicle of Janus $\mathrm{Au}-\mathrm{MnO}$ Nanoparticles for Promoted Tumor Penetration and Sono-Chemodynamic Therapy of Orthotopic Liver Cancer. Angew Chem Int Ed Engl. 2020;59(4):1682-1688. doi:10.1002/anie.201912768

27. Wang Y, Xie Y, Li J, et al. Tumor-Penetrating Nanoparticles for Enhanced Anticancer Activity of Combined Photodynamic and Hypoxia-Activated Therapy. ACS Nano. 2017;11(2):2227-2238. doi:10.1021/acsnano.6b08731

28. He X, Chen X, Liu L, et al. Sequentially Triggered Nanoparticles with Tumor Penetration and Intelligent Drug Release for Pancreatic Cancer Therapy. Adv Sci. 2018;5(5):1701070. doi:10.1002/advs.201701070

29. Kim J, Jo C, Lim WG, et al. Programmed Nanoparticle-Loaded Nanoparticles for Deep-Penetrating 3D Cancer Therapy. Adv Mater. 2018;30(29):e1707557. doi:10.1002/adma.201707557

30. Manzoor AA, Lindner LH, Landon CD, et al. Overcoming limitations in nanoparticle drug delivery: triggered, intravascular release to improve drug penetration into tumors. Cancer Res. 2012;72 (21):5566-5575. doi:10.1158/0008-5472.CAN-12-1683

31. Martin JD, Panagi M, Wang C, et al. Dexamethasone Increases cisplatin-loaded nanocarrier delivery and efficacy in metastatic breast cancer by normalizing the tumor microenvironment. ACS Nano. 2019;13(6):6396-6408. doi:10.1021/acsnano.8b07865

32. Tannock I, Tunggal JK. Limited Penetration of Anticancer Drugs through Tumor Tissue: a Potential Cause of Resistance of Solid Tumors to Chemotherapy. Clin Cancer Res. 2002;8(3):878-884.

33. Peterson TE, Kirkpatrick ND, Huang Y, et al. Dual inhibition of Ang-2 and VEGF receptors normalizes tumor vasculature and prolongs survival in glioblastoma by altering macrophages. Proc Natl Acad Sci US A. 2016;113(16):4470-4475. doi:10.1073/pnas.1525349113

34. Jain RK. Normalization of tumor vasculature: an emerging concept in antiangiogenic therapy. Science. 2005;307(5706):58-62. doi:10.1126/ science. 1104819

35. Carmeliet P, Jain RK. Principles and mechanisms of vessel normalization for cancer and other angiogenic diseases. Nat Rev Drug Discov. 2011;10(6):417-427. doi:10.1038/nrd3455

36. Virag R, Frydman D, Legman M, Virag H. Intracavernous Injection of Papaverine as a Diagnostic and Therapeutic Method in Erectile Failure. Angiology. 1984;35(2):79-87. doi:10.1177/000331978403500203

37. Juenemann K-P, Lue TF, Fournier GR, Tanagho EA. Hemodynamics of papaverine- and phentolamine-induced penile erection. J Urol. 1986;136(1):158-161. doi:10.1016/S0022-5347(17)44763-X

38. Benej M, Hong X, Vibhute S, et al. Papaverine and its derivatives radiosensitize solid tumors by inhibiting mitochondrial metabolism. Proc Natl Acad Sci U S A. 2018;115(42):10756-10761. doi:10.1073/ pnas. 1808945115
39. Triner L, Vulliemoz Y, Schwartz I, Nahas GG. Cyclic phosphodiesterase activity and the action of papaverine. Biochem Biophys Res Commun. 1970;40(1):64-69. doi:10.1016/0006-291X(70)91046-6

40. Li H, Zhu X, Yao G, Wang Z. Papaverine improves sublingual blood flow in patients with septic shock. J Surg Res. 2015;195(1):271-276. doi:10.1016/j.jss.2015.01.016

41. Hu CM, Zhang L, Aryal S, Cheung C, Fang RH, Zhang L. Erythrocyte membrane-camouflaged polymeric nanoparticles as a biomimetic delivery platform. Proc Natl Acad Sci U S A. 2011;108(27):10980-10985. doi:10.1073/pnas.1106634108

42. Gao C, Lin Z, Wang D, Wu Z, Xie H, He Q. Red blood cell-mimicking micromotor for active photodynamic cancer therapy. ACS Appl Mater Interfaces. 2019;11(26):23392-23400. doi:10.1021/ acsami.9b07979

43. Wang Y, Zhang K, Qin X, et al. Biomimetic nanotherapies: red blood cell based core-shell structured nanocomplexes for atherosclerosis management. Adv Sci. 2019;6(12):1900172. doi:10.1002/advs.2019 00172

44. Shao J, Pijpers IAB, Cao S, et al. Biomorphic engineering of multifunctional polylactide stomatocytes toward therapeutic nano-red blood cells. Adv Sci. 2019;6(5):1801678. doi:10.1002/advs.2018 01678

45. Hu C-MJ, Fang RH, Wang K-C, et al. Nanoparticle biointerfacing by platelet membrane cloaking. Nature. 2015;526(7571):118-121. doi:10.1038/nature 15373

46. Zhang P, Liu G, Chen X. Nanobiotechnology: cell Membrane-Based Delivery Systems. Nano Today. 2017;13:7-9. doi:10.1016/j.nantod. 2016.10.008

47. Zhen X, Cheng P, Pu K. Recent advances in cell membrane-camouflaged nanoparticles for cancer phototherapy. Small. 2019;15(1):e1804105. doi:10.1002/smll.201804105

48. Song G, Chen Y, Liang C, et al. Catalase-Loaded TaOx Nanoshells as Bio-Nanoreactors Combining High-Z Element and Enzyme Delivery for Enhancing Radiotherapy. Adv Mater. 2016;28(33):7143-7148. doi:10.1002/adma.201602111

49. Zhu DM, Xie W, Xiao YS, et al. Erythrocyte membrane-coated gold nanocages for targeted photothermal and chemical cancer therapy. Nanotechnology. 2018;29(8):084002. doi:10.1088/1361-6528/aa9ca1

50. Barker HE, Paget JT, Khan AA, Harrington KJ. The tumour microenvironment after radiotherapy: mechanisms of resistance and recurrence. Nat Rev Cancer. 2015;15(7):409-425. doi:10.1038/ $\operatorname{nrc} 3958$

51. Pries TS, Gaehtgens P. Biophysical aspects of blood flow in the microvasculature. Cardiovasc Res. 1996;32(4):654-667. doi:10.101 6/S0008-6363(96)00065-X
International Journal of Nanomedicine

\section{Publish your work in this journal}

The International Journal of Nanomedicine is an international, peerreviewed journal focusing on the application of nanotechnology in diagnostics, therapeutics, and drug delivery systems throughout the biomedical field. This journal is indexed on PubMed Central, MedLine, CAS, SciSearch ${ }^{\mathbb{R}}$, Current Contents ${ }^{\mathbb{B}} /$ Clinical Medicine,
Journal Citation Reports/Science Edition, EMBase, Scopus and the Elsevier Bibliographic databases. The manuscript management system is completely online and includes a very quick and fair peer-review system, which is all easy to use. Visit http://www.dovepress.com/ testimonials.php to read real quotes from published authors. 